\title{
Expression of long noncoding RNAs in the ovarian granulosa cells of women with diminished ovarian reserve using high-throughput sequencing
}

\section{Li Dong}

Shandong University of Traditional Chinese Medicine

\section{Xin Xin}

Shandong University of Traditional Chinese Medicine

\section{Hsun-Ming Chang}

University of British Columbia

\section{Peter C.K. Leung}

University of British Columbia

\section{Chen Yu}

Shandong University of Traditional Chinese Medicine

\section{Fang Lian}

Affiliated Hospital of Shandong University of Traditional Chinese Medicine

Haicui Wu ( $\nabla$ haicui_w@163.com )

Affiliated Hospital of Shandong University of Traditional Chinese Medicine

\section{Research Article}

Keywords: Diminished ovary reserve, Ovarian granulosa cells, Long non-coding RNA, High-throughput sequencing

Posted Date: May 23rd, 2022

DOI: https://doi.org/10.21203/rs.3.rs-1276715/v2

License: (c) (1) This work is licensed under a Creative Commons Attribution 4.0 International License. Read Full License 


\section{Abstract \\ Background}

Infertility is a global reproductive-health problem, and diminished ovarian reserve (DOR) is one of the common causes of female infertility. Long noncoding RNAs (IncRNAs) are crucial regulators of numerous physiological and pathological processes in humans. However, whether IncRNAs are involved in the development of DOR remains to be elucidated.

\section{Methods}

Ovarian granulosa cells (OGCs) extracted from infertile women with DOR and from women with normal ovarian reserve (NOR) were subjected to high-throughput sequencing. Comprehensive bioinformatics analysis was conducted to identify the differential expression of messenger RNAs (mRNAs) and IncRNAs. Sequencing results were validated by the selection of random IncRNAs using real-time reverse transcription-quantitative polymerase chain reaction.

\section{Results}

Compared with the NOR group, a total of 244 IncRNAs were upregulated (53 known and 191 novel), and 222 IncRNAs were downregulated (36 known and 186 novel) in the DOR group. Similarly, 457 mRNAs had differential expression between the two groups. Of these, 169 were upregulated and 288 were downregulated. Bioinformatics analysis revealed that the differentially expressed genes of mRNA and IncRNAs were considerably enriched in "cell adhesion and apoptosis", "steroid biosynthesis", and "immune system". A co-expression network comprising IncRNAs and their predicted target genes revealed the possible involvement of the "thyroid hormone signaling pathway" and "protein binding, digestion and absorption" in DOR pathogenesis. The expression of SLC16A10 was positively regulated by multiple IncRNAs. Seven differentially expressed IncRNAs (NEAT1, RAB5A, MEG3, GNG12, MST1L, GCN1, and ZEB2-AS1) were identified, among which the expression of NEAT1, GNG12 and ZEB2-AS1 was in accordance with RNA-sequencing.

\section{Conclusions}

We presented the first data showing that the expression profiles of IncRNA and mRNA in OGCs between NOR and DOR patients using RNA sequencing. The IncRNAs that we identified may serve as novel diagnostic biomarkers for patients with DOR.

\section{Background}


Infertility is a worldwide problem involving couples of reproductive ages. It has become the third most common disease after cancer and cardiovascular diseases [1]. As a serious medical problem, infertility can have destructive effects on the quality of life and marital satisfaction. As economic and social pressures increase, the age at which women have children continues to increase. The proportion of infertile women due to diminished ovarian reserve (DOR) is increasing gradually worldwide. The term of DOR was first described by Navot and colleagues in 1987 [2]. It is characterized by a decline in the quantity and/or quality of oocytes, with increased serum levels of follicle-stimulating hormone (FSH) and decreased serum levels of anti-Müllerian hormone (AMH) [3]. The main clinical manifestations are irregular menstruation, perimenopause syndrome, and infertility. The United States Assisted Reproductive Technology Population Survey revealed an increase in DOR prevalence from 19\% in 2004 up to $26 \%$ in 2011 [3]. Studies have demonstrated that DOR typically develops into premature ovarian failure (POF) in $1-6$ years [4].

Women with DOR usually result in poor pregnancy outcomes and present major challenges in reproductive medicine, particularly receiving in vitro fertilization/intracytoplasmic sperm injection (IVF/ICSI) treatment [5]. However, the etiology and pathogenesis of DOR are not clear. The key etiological factors are age, genetics, iatrogenicity (surgery, radiotherapy, or chemotherapy), and immune and environmental factors. Therefore, to improve pregnancy outcomes in women with DOR, the clinicians should focus on the molecular mechanisms underlying DOR occurrence. In this way, timely prediction of the diagnosis, necessary interventions, and therapeutic measures through identification of clinical biomarkers can be undertaken.

Long noncoding RNAs (IncRNAs) are functional RNA molecules of $>200$ nucleotides in length that cannot be translated into proteins. Recently, studies have revealed that IncRNAs regulate the expression of genes in terms of transcriptional, posttranscriptional, and epigenetic modifications [6]. These actions may affect the growth, development, aging, and disease progression of organisms [7]. Additionally, IncRNAs are involved in the regulation of stem cell differentiation, embryonic development, cell proliferation, apoptosis, cell metabolism, and immune reaction [8, 9]. Recent studies have shown differential expression of IncRNAs in several gynecological disorders, such as endometriosis [10], premature ovarian insufficiency (POI) [11], and polycystic ovary syndrome (PCOS) [12]. Although several IncRNAs have been found to be involved in the abovementioned ovarian diseases, the association between IncRNAs and DOR is largely unknown.

Technologies based on transcription, gene chips, and high-throughput sequencing, have found that various IncRNAs are related to the occurrence, development, and treatment of diseases. The use of biological methods to study differences in the expression of IncRNAs between patients and healthy individuals and prescreening IncRNAs by high-throughput sequencing can aid in the discovery of new targets related to many diseases. In-depth study of the biomolecular mechanisms of IncRNAs can help explain the complexity and pathological states of diseases and support the diagnosis and treatment of refractory diseases. 
In this study, we aimed to employ transcriptome analysis to investigate the biological role of IncRNAs during DOR progression. Ovarian granulosa cells (OGCs) from women with DOR and those from women with normal ovarian reserve (NOR) were obtained to perform RNA-sequencing (RNA-seq) and analyze the expression profiles between two groups. Bioinformatics analysis was undertaken to identify differentially expressed (DE) IncRNAs and messenger (m)RNAs and their associated functions and signaling pathways. In addition, we applied real-time reverse transcription-quantitative polymerase chain reaction (RT-qPCR) to verify the differential expression of seven IncRNAs. These aberrantly expressed IncRNAs may serve as potential biomarkers for the development of DOR and may introduce new routes for the diagnosis of women with POF.

\section{Results}

\section{Baseline characteristics of patients used for sequencing}

As presented in Table 1, patients in the DOR group had low AMH levels, low antral follicle count (AFC), low number of oocytes obtained and high basal FSH levels compared to the NOR group, the differences in the above characteristics being indicators of ovarian reserve $(P<0.001)$. No other features were significantly different $(P>0.05)$. 
Table 1

Comparison of the baseline characteristics between the NOR group and DOR group used for sequencing

\begin{tabular}{|llll|}
\hline & NOR $(\mathbf{n}=6)$ & DOR $(\mathbf{n}=6)$ & P \\
\hline Patient age (years) & $30.50 \pm 1.76$ & $35.17 \pm 4.88$ & 0.068 \\
\hline Infertility duration (years) & $4.50 \pm 2.95$ & $5.17 \pm 2.40$ & 0.677 \\
\hline Infertility type (\%) & & & 0.567 \\
\hline Primary & $4 / 6(66.7)$ & $2 / 6(33.3)$ & \\
\hline Secondary & $2 / 6(33.3)$ & $4 / 6(66.6)$ & 0.181 \\
\hline BMI (kg/m ${ }^{2}$ ) & $22.72 \pm 0.98$ & $21.75 \pm 1.33$ & $<0.001$ \\
\hline AMH (ng/mL) & $4.64 \pm 1.11$ & $0.79 \pm 0.23$ & $<0.001$ \\
\hline Basal FSH (mIU/mL) & $6.99 \pm 1.40$ & $13.57 \pm 2.04$ & $<0.001$ \\
\hline AFC (n) & $25.67 \pm 2.73$ & $6.83 \pm 1.94$ & 0.135 \\
\hline Previous IVF/ICSI attempts (n) & $0.00 \pm 0.00$ & $1.17 \pm 1.60$ & $<0.001$ \\
\hline Number of oocytes retrieved & $16.17 \pm 3.49$ & $4.00 \pm 2.28$ & \\
\hline Data are the mean \pm SD and proportion (\%). & & & \\
\hline $\begin{array}{l}\text { AMH, anti-Müllerian hormone; AFC, antral follicle count; BMI, body mass index; FSH, follicle- } \\
\text { stimulating hormone; ICSI, intracytoplasmic sperm injection; IVF, in vitro fertilization. }\end{array}$ & \\
\hline
\end{tabular}

\section{Sequencing of complementary (c)DNA libraries and transcriptome profiles of OGCs}

A total of $999,067,624$ raw reads were acquired using the NovaSeq ${ }^{\text {TM }} 6000$ platform (Illumina, San Diego, CA, USA). After excluding adapter contamination, undetermined bases, and low-quality bases, $902,773,866$ clean reads were obtained, accounting for 135.42 GB. Although the GC content of the clean data was $~ 42 \%$, the quality scores of clean reads were $>99.9$ and $>98.83 \%$ for Q20 and Q30, respectively. In summary, these results indicated that the reliability and quality of the sequencing data were sufficient for further analyses (Table 2). Over $96 \%$ of clean reads were mapped to the reference human genome using HISAT (http://daehwankimlab.github.io/hisat2/), including uniquely mapped reads from $84.10-86.76 \%$ (Supplemental Table 1). 
Table 2

Statistical data of the reads for 12 cDNA libraries

\begin{tabular}{|llllllllll|}
\hline Sample & $\begin{array}{l}\text { Raw } \\
\text { reads }\end{array}$ & $\begin{array}{l}\text { Raw } \\
\text { bases }\end{array}$ & $\begin{array}{l}\text { Clean } \\
\text { reads }\end{array}$ & $\begin{array}{l}\text { Clean } \\
\text { bases }\end{array}$ & $\begin{array}{l}\text { Valid ratio } \\
\text { (reads) }\end{array}$ & Q20\% & Q30\% & $\begin{array}{l}\text { GC } \\
\text { content } \\
(\%)\end{array}$ \\
\hline DOR_1 & 95746384 & $14.36 \mathrm{G}$ & 87242788 & $13.09 \mathrm{G}$ & 91.12 & 99.99 & 98.96 & 42 \\
\hline DOR_2 & 90791244 & $13.62 \mathrm{G}$ & 79121838 & $11.87 \mathrm{G}$ & 87.15 & 99.99 & 98.88 & 43 \\
\hline DOR_3 & 72114510 & $10.82 \mathrm{G}$ & 66912764 & $10.04 \mathrm{G}$ & 92.79 & 99.99 & 98.96 & 41.50 \\
\hline DOR_4 & 70676974 & $10.60 \mathrm{G}$ & 65727486 & $9.86 \mathrm{G}$ & 93.00 & 99.99 & 98.97 & 41 \\
\hline DOR_5 & 91948332 & $13.79 \mathrm{G}$ & 85217766 & $12.78 \mathrm{G}$ & 92.68 & 99.99 & 98.93 & 42 \\
\hline DOR_6 & 81704846 & $12.26 \mathrm{G}$ & 74061172 & $11.11 \mathrm{G}$ & 90.64 & 99.99 & 98.83 & 44 \\
\hline NOR_1 & 88597962 & $13.29 \mathrm{G}$ & 81949398 & $12.29 \mathrm{G}$ & 92.50 & 99.99 & 98.91 & 42 \\
\hline NOR_2 & 67940108 & $10.19 \mathrm{G}$ & 62960406 & $9.44 \mathrm{G}$ & 92.67 & 99.99 & 98.93 & 41.50 \\
\hline NOR_3 & 66981682 & $10.05 \mathrm{G}$ & 61871236 & $9.28 \mathrm{G}$ & 92.37 & 99.99 & 98.98 & 42 \\
\hline NOR_4 & 88814944 & $13.32 \mathrm{G}$ & 73435164 & $11.02 \mathrm{G}$ & 82.68 & 99.99 & 98.91 & 43.50 \\
\hline NOR_5 & 90275194 & $13.54 \mathrm{G}$ & 77710494 & $11.66 \mathrm{G}$ & 86.08 & 99.99 & 98.88 & 43 \\
\hline NOR_6 & 93475444 & $14.02 \mathrm{G}$ & 86563354 & $12.98 \mathrm{G}$ & 92.61 & 99.99 & 98.97 & 42 \\
\hline
\end{tabular}

\section{Identification of IncRNAs and mRNAs}

To further recognize the protein-coding ability of unknown transcripts, Coding Potential Calculator (CPC; http://cpc2.gao-lab.org/) and Coding-Non-Coding Index (CNCl; https://github.com/www-bioinfoorg/CNCl/) were designed to exclude those with coding potential. As a result, a total of 45,333 novel IncRNAs were detected from 12 cDNA libraries (Supplemental Table 2). These IncRNAs were dispersed evenly across the 46 human chromosomes (Fig. 1a). With regard to the genomic location of the IncRNAs, the types of IncRNAs were class_code "i" (intraintronic transcript, 72.24\%), class_code "u" (intergenic transcript, 18.37\%), class_code "o" (sense transcript, 3.55\%), class_code "j" (bidirectional transcript, 3.25\%), and class_code " $x$ " (antisense transcript, 2.59\%). These IncRNAs showed no apparent bias for genomic location (Supplemental Fig. 1 and Fig. 1b). Additionally, a total of 47,520 known IncRNAs were identified between the two groups.

We compared IncRNAs with mRNAs to illustrate the structural features of IncRNAs. The expression and number of IncRNAs were comparable to those of mRNAs (Fig. 1C). Furthermore, the median length of IncRNA transcripts was $1504 \mathrm{bp}$, which was shorter than the median length of $2317 \mathrm{bp}$ for mRNA transcripts. These findings suggest that these IncRNAs are shorter than the mRNAs (Fig. 1d). There were fewer exons in IncRNAs (mean, 3) than in mRNAs (mean, 9). In total, $68.86 \%$ of mRNAs had $\geq 5$ exons, 
whereas $77.02 \%$ of IncRNAs had $\leq 3$ exons (Fig. 1e). Moreover, in OGCs, the predicted open reading frame (ORF) length of IncRNAs was shorter than that of mRNAs (Fig. 1f).

\section{Expression profile of mRNAs}

A total of 457 DE mRNA genes were found between the OGCs of the two groups. Of these genes, 169 genes were upregulated, and 288 genes were downregulated (Fig. 2a-b). To further explore the functions of these DE mRNA genes, analyses of functional enrichment were performed using the Gene Ontology (G0) (http://geneontology.org/) database. Among the 457 DE mRNA genes, $3404 \mathrm{GO}$ terms with functional information were enriched (Fig. 2c-d). A total of $542 \mathrm{GO}$ terms were enriched significantly $(P<$ 0.05). For biological process (BP), 359 terms were found, including "cell adhesion" (G0:0007155), "positive regulation of apoptotic process" (G0:0043065) and "steroid biosynthetic process" (G0:0006694). For molecular function (MF), 137 terms were discovered, including "oxidoreductase activity" (G0:0016491). For "cellular component" (CC), 42 terms were found, including "endoplasmic reticulum lumen” (G0:0005788) (Supplemental Table 3).

We used the Kyoto Encyclopedia of Genes and Genomes (KEGG) (www.genome.jp/kegg/) database to identify which genes had significantly enriched signaling pathways. Nineteen significantly enriched signaling pathways related to the DE mRNA genes $(P<0.05)$ were found, including "steroid biosynthesis", "focal adhesion", "ECM-receptor interaction", "PPAR signaling pathway", and "PI3K-AKT signaling pathway" (Fig. 2e).

\section{Expression profile of IncRNAs}

We discovered that 466 IncRNAs had differential expression between the DOR group and NOR group. Of these, 244 were upregulated (53 known and 191 novel), and 222 were downregulated (36 known and 186 novel) (Supplemental Table 4, Fig. 3a-b). To further probe the functions of these IncRNAs, we forecasted the cis-regulated target genes of the DE IncRNAs between the DOR group and NOR group. A total of 52 IncRNAs were found to have target genes if 100 kilobase pairs (kbp) was used as the cutoff. Some IncRNAs had 2-3 target genes, and 57 probable IncRNA target genes were identified (Supplemental Table 5). Based on these cis-regulated target genes, we used the GO database to identify 76 important GO terms: 57 BP terms (e.g., "stem cell proliferation, GO:0072089), 6 CC terms (e.g., "proteasome activator complex", G0:0008537), and 13 MF terms (e.g., "CXCR3 chemokine receptor binding”, G0:0048248) (Fig. 3c-d). Analyses of signaling pathway enrichment using the KEGG database revealed that these IncRNA target genes were primarily enriched in "ferroptosis", "fatty acid biosynthesis", and the "JAK-STAT signaling pathway" (Fig. 3e).

We discovered the predicted outcomes of the DE IncRNAs with cis-regulated genes. We listed the first-six and last-four IncRNA-gene pairs according to the Pearson correlation coefficient. The first-six IncRNAgene pairs were regulated in the same direction, whereas the last-four IncRNA-gene pairs were in the opposite direction (Table 3). 
Table 3

Differentially expressed IncRNA-gene pairs between the DOR group and NOR group.

\begin{tabular}{|llll|}
\hline Gene & IncRNA transcript & Cis location (bp) & Pearson correlation coefficient \\
\hline CXCL10 & MSTRG.57192.1 & $1 \mathrm{~K}$ & 1.00 \\
\hline TNFSF15 & MSTRG.77544.1 & 100K & 1.00 \\
\hline PGBD5 & MSTRG.9312.2 & 100K & 1.00 \\
\hline CNTN3 & MSTRG.52583.1 & 100K & 0.99 \\
\hline SLC16A10 & MSTRG.66173.1 & 10K & 0.99 \\
\hline SLC16A10 & MSTRG.66167.1 & 100K & 0.99 \\
\hline BNIP2 & MSTRG.27592.2 & 100K & -0.31 \\
\hline SYNCRIP & ENST00000656092 & 100K & -0.32 \\
\hline NPIPB15 & MSTRG.31257.1 & 10K & -0.33 \\
\hline C2CD2 & MSTRG.48668.1 & 100K & -0.35 \\
\hline
\end{tabular}

\section{Coenriched $\mathrm{GO}$ terms of the DE IncRNAs and mRNAs}

We then aimed to identify the key pathways that regulate the ovarian reserve. Hence, we identified five significantly enriched GO terms in the enrichment of the DE mRNAs and the enrichment of the IncRNA target genes. The significantly enriched GO terms were "CXCR chemokine receptor binding", "CXCR3 chemokine receptor binding", "positive regulation of transforming growth factor-beta production", "regulation of bile acid biosynthetic processes", and "cellular response to organonitrogen compound". Three pathways were involved in BP. and the other two pathways were involved in MF (Table 4).

Table 4

Coenriched GO terms of DE IncRNAs and mRNAs.

\begin{tabular}{|llc|}
\hline GO Term & Go function & P \\
\hline G0:0048248 (CXCR3 chemokine receptor binding) & molecular_function & 0.002 \\
\hline $\begin{array}{l}\text { G0:0071636 (positive regulation of transforming growth factor beta } \\
\text { production) }\end{array}$ & biological_process & 0.005 \\
\hline G0:0045236 (CXCR chemokine receptor binding) & molecular_function & 0.010 \\
\hline G0:0070857 (regulation of bile acid biosynthetic process) & biological_process & 0.027 \\
\hline Go:0071417 (cellular response to organonitrogen compound) & biological_process & 0.034 \\
\hline $\begin{array}{l}\text { Construction Of a CO-expression netwOrk Of DE InCRNAS and } \\
\text { MRNAs }\end{array}$ & \\
\hline
\end{tabular}


We performed comprehensive analyses of the IncRNA-mRNA regulatory network to further explore the potential regulatory mechanism. Co-expression analyses between the DE mRNA genes and IncRNAs were performed first. Twenty-four positively correlated IncRNA-mRNA co-expression pairs containing $24 \mathrm{DE}$ IncRNAs and 19 DE mRNAs were acquired at the verge of a Pearson correlation coefficient $(r>0.40)$ (Supplemental Table 6). Then, a IncRNA-mRNA regulatory network comprising dysregulated IncRNAs and their cis-target mRNAs was visualized using Cytoscape v3.7.1 (https://cytoscape.org/). Solute carrier family 16 member 10 (SLC16A10) was included in this network and was regulated by five IncRNAs: MSTRG.66173, MSTRG.66167, MSTRG.66177, MSTRG.66174, and MSTRG.66170 (Fig. 4). Among them, SLC16A10 was enriched in several GO terms, including "protein binding" (G0:0005515), "thyroid hormone generation" (GO:0006590), and "cell junction" (G0:0030054). SLC16A10 was also enriched in the "thyroid hormone signaling pathway" and "protein digestion and absorption".

\section{Verification of the DE IncRNAs by RT-qPCR}

To verify the RNA-seq results, RT-qPCR was performed to examine seven of the identified DE IncRNAs between the OGCs from the DOR group and NOR group. Table 5 displayed the baseline characteristics of the case used for RT-qPCR validation, with patients in the DOR group not significantly different from those in the NOR group ( $P>0.05)$, except for indicators related to ovarian reserve such as AMH level, AFC, and number of oocytes retrieved $(P<0.05)$. These seven IncRNAs include nuclear rich abundant transcript 1 (NEAT1), Ras-related protein in brain 5A (RAB5A), maternally expressed gene 3 (MEG3), guanine nucleotide-binding protein subunit gamma-12 (GNG12), macrophage stimulating 1 like (MST1L), general control nonderepressible 1 (GCN1), zinc-finger E-box binding homeobox 2-AS1(ZEB2-AS1). Table 6 presented information on the relevant sequencing results for the above IncRNAs. 
Table 5

Comparison of the baseline characteristics between the NOR group and DOR group used for RT-qPCR

\begin{tabular}{|llll|}
\hline & NOR $(\mathbf{n}=6)$ & DOR $(\mathbf{n}=6)$ & P \\
\hline Patient age (years) & $30.50 \pm 2.88$ & $35.50 \pm 4.72$ & 0.057 \\
\hline Infertility duration (years) & $3.00 \pm 1.67$ & $3.50 \pm 2.43$ & 0.687 \\
\hline Infertility type (\%) & & & 1.000 \\
\hline Primary & $2 / 6(33.3)$ & $1 / 6(16.7)$ & \\
\hline Secondary & $4 / 6(66.7)$ & $5 / 6(83.3)$ & \\
\hline BMI (kg/m $\left.{ }^{2}\right)$ & $20.20 \pm 2.61$ & $21.52 \pm 2.86$ & 0.424 \\
\hline AMH (ng/mL) & $4.13 \pm 0.59$ & $0.78 \pm 0.22$ & $<0.001$ \\
\hline Basal FSH (mIU/mL) & $6.41 \pm 1.48$ & $11.02 \pm 4.42$ & 0.051 \\
\hline AFC (n) & $26.00 \pm 2.68$ & $7.00 \pm 2.10$ & $<0.001$ \\
\hline Previous IVF/ICSI attempts (n) & $0.67 \pm 1.21$ & $0.67 \pm 0.82$ & 1.000 \\
\hline Number of oocytes retrieved & $16.50 \pm 7.79$ & $4.17 \pm 1.84$ & 0.011 \\
\hline Data are the mean \pm SD and proportion (\%). & & & \\
\hline $\begin{array}{l}\text { AMH, anti-Müllerian hormone; AFC, antral follicle count; BMI, body mass index; FSH, follicle- } \\
\text { stimulating hormone; ICSI, intracytoplasmic sperm injection; IVF, in vitro fertilization. }\end{array}$ & \\
\hline
\end{tabular}

Table 6

Sequencing information of IncRNAs used for RT-qPCR verification

\begin{tabular}{|lllll|}
\hline Transcript name & Gene name & $\log _{\mathbf{2}}$ (fold_change) & Regulation & P \\
\hline MSTRG.15237.9 & NEAT1 & -4.69 & Down & 0.041834004 \\
\hline MSTRG.50996.10 & RAB5A & -3.07 & Down & 0.003518952 \\
\hline MSTRG.25915 & MEG3 & -3.83 & Down & 0.176775112 \\
\hline MSTRG.5280.1 & GNG12 & -4.85 & Down & 0.0003983567 \\
\hline MSTRG.3657.2 & MST1L & -4.88 & Down & 0.000306066 \\
\hline MSTRG.20933.1 & GCN1 & 5.98 & Up & 0.000182295 \\
\hline MSTRG.43755 & ZEB2-AS1 & -13.50 & Down & 0.000166045 \\
\hline
\end{tabular}

Of these, the expression of NEAT1, GNG12, and ZEB2-AS1 was consistent with the RNA-seq results. The expression of the remaining four IncRNAs was not significantly different but showed a consistent trend 
with the RNA-seq data (Fig. 5). These results indicate that the seq-RNA data were reliable.

\section{Discussion}

Follicular reserve and ovarian function are measures of reproductive endocrine functionality and fertility potential [13]. OGCs play important roles in the regulation of follicle activation, growth, development, and atresia [14]. Recently, several studies targeting specific genes and regulating various signaling pathways have shown that IncRNAs have vital roles in the formation and development of ovarian follicles [15-17].

We report, for the first time, the expression profiles of IncRNAs and mRNAs in OGCs of DOR patients and NOR patients, as identified by high-throughput sequencing. We identified 466 DE IncRNAs and 457 mRNAs associated with ovarian reserve. We showed that these dysregulated genes were involved in multiple biological processes, including cell adhesion and apoptosis, the immune system, and signaling pathways such as steroid biosynthesis. Notably, multiple IncRNAs could contribute to DOR by targeting and regulating signaling pathways mediated by SLC16A10, such as thyroid hormone synthesis and protein digestion and absorption. Overall, our results indicate the potential regulatory role of related IncRNAs and mRNAs in DOR.

In recent years, several studies have identified an important role for IncRNAs in the regulation of follicle development $[18,19]$. Studies have identified differential expression of IncRNAs associated with reproductive function by using RNA-seq of OGCs obtained from humans [20], pigs [21], and chickens [22]. Most of those studies have focused on PCOS [23], endometriosis [24, 25], and POF [26, 27]. The IncRNAs we identified had fewer exons, shorter ORFs, and shorter transcript lengths compared with those in protein-coding transcripts, and these characteristics were similar to those from porcine OGCs [28] and human placentas at term [29]. These data demonstrate that the IncRNA outcomes we obtained were reliable.

Several research teams have reported aberrant IncRNA expression in POI and identified hundreds of IncRNAs associated with POI. Some of these IncRNAs could be used as biomarkers for the diagnosis or prognosis of DOR $[16,30]$. The discrepancies between the DE IncRNAs identified in our study and those in other studies are due (at least in part) to differences in sample selection and sequencing methods. Moreover, several researchers have conducted functional studies on these DE IncRNAs [31-33] and reported different underlying molecular mechanisms. However, most of those studies concentrated on a particular gene or signaling pathway and did not analyze them from a holistic perspective. Additionally, most of the identified IncRNAs and mRNAs were associated with proliferative and apoptotic processes. The DE genes identified in our study were mostly involved in the signaling pathways related to the adhesion, proliferation, and apoptosis of cells, steroid biosynthesis and metabolism, and the immune response. All of these are biological processes involved in follicular atresia induced by OGC apoptosis $[34,35]$.

Folliculogenesis is regulated by multiple mechanisms involving endocrine and intraovarian signaling pathways [36,37], and changes caused by the differential expression of genes may further affect ovarian 
reserve. We revealed several DE IncRNAs that could be associated with ovarian reserve (NEAT1, MEG3, GNG12, MST1L, RAB5A, and ZEB2-AS1), all of which have vital roles in the proliferation and apoptosis of cells and merit further investigation. Among these IncRNAs, NEAT1 is a relatively well-studied IncRNA involved in the formation and maintenance of a "nuclear paraspeckle" (a nuclear body with multiple geneexpression functions). NEAT1 can promote disease through regulation of mitochondrial function [38]. NEAT1 is expressed abundantly in oocytes as well as in primordial, primary, and small antral follicles. It is involved mainly in the expression of genes related to apoptosis and extracellular matrix-related functions to achieve epigenetic control of follicular development $[39,40]$. Interference with NEAT1 alleviates the pathological changes of PCOS by reducing the expression level of insulin-like growth factor 1 . Therefore, it suppresses OGC apoptosis and enhances cell proliferation [41]. Some studies have analyzed publicly available RNA-seq data and found that NEAT1 and MEG3 (which are expressed abundantly in oocytes) are essential for oocyte growth [39], which we also discovered in this study. MEG3 can inhibit the proliferation of OGCs and POF through the p53-p66Shc pathway [15], which differs from our findings. Therefore, further studies are needed to confirm the mechanism of action of MEG3 in oocytes. RAB5Apositive vesicles modulate the localization and levels of centromere protein $\mathrm{F}$ at centromeres, likely through interaction with nuclear lamina. Therefore, an appropriate spindle length and kineto-microtubule attachment are ensured in meiotic oocytes [42]. GNG12 [43] and ZEB2-AS1 [44, 45] can promote the growth of tumor cells by regulating the proliferation, apoptosis, and migration of cells. MST1L [46] can regulate disease development through an inflammatory response pathway. GNG12, ZEB2-AS1, and MST1L tended to have low expression in the OGCs from DOR patients. Such low expression level may contribute to DOR development by increasing apoptosis and inhibiting cell proliferation in OGCs. In particular, GNG12 and ZEB2-AS1 have been insufficiently investigated with respect to follicle growth. However, we observed significant downregulation of GNG12 and ZEB2-AS1 expression by RNA-seq and RT-qPCR, suggesting that GNG12 and ZEB2-AS1 may be closely related to DOR function.

We assessed the enrichment of functions and signaling pathways using the GO database and KEGG database, respectively, to reveal the biological functions of the DE IncRNA target genes and mRNAs. The DE genes were involved mainly in cell adhesion, steroid biosynthesis, and pathways related to immunity and apoptosis, which are the functional pathways of OGCs [34,47,48]. Cell adhesion is involved in the in vitro culture of porcine [49] and human [50] OGCs and can affect the proliferative potential and survival capability. Pashaiasl and collaborators measured the mRNA expression profile of the OGCs of DOR patients. Similar to our findings, they identified DE genes involved in focal adhesion [51], as well as DE genes involved in ossification, ovarian-follicle development, vasculogenesis, and sequence-specific activity of DNA-binding transcription factors. OGCs are the most important somatic cells for the synthesis of steroid hormones. We found that the DE genes could affect steroid biosynthesis and thus regulate the proliferation and apoptosis of OGCs through multiple pathways. These findings have been documented in studies in sheep [52], mice [53] and women with POF [54].

Autoimmunity is responsible for approximately $4-30 \%$ of $\mathrm{POI}$ cases [55]. Women suffering from POI carry a high risk of having autoimmune disorders [56]. Chemokines play an important role in reproductive immunology [57] and regulate leukocyte migration by attracting cells that express their cognate ligands. 
C-X-C motif chemokine ligand 12 (CXCL12), C-X-C motif chemokine receptor 4 (CXCR4), and CXCR7 can influence the development of endometriosis, Asherman's syndrome, endometrial cancer, and ovarian cancer [58]. CXCR3, which was identified in the present study, has been investigated less thoroughly. Therefore, women should be screened for common autoantibodies (e.g., steroid cell, anti-ovarian, and anti-thyroid autoantibodies), which can aid in the early diagnosis and treatment of DOR and the prevention of POF. A prospective cohort study involving 164 women found a higher prevalence of OGC apoptosis, worse ovarian response, lower oocyte count, lower embryo count, and poor pregnancy outcomes in patients with DOR [59].

We found that the phosphoinositide 3-kinase/protein kinase B (PI3K-AKT) and Janus kinase/signal transducer and activator of transcription (JAK-STAT) pathways enriched by the DE genes were associated with apoptotic processes. Several studies have demonstrated that the inhibition of the PI3K-AKT pathway leads to apoptosis and autophagy in OGCs $[53,54]$. The importance of the JAK/STAT signaling pathway in ovarian development and folliculogenesis in horses [60] and humans [61] has been demonstrated. Polyfluoroalkyl substances reduce the ovarian reserve and decrease endogenous hormone synthesis by activating the peroxisome proliferator-activated receptor (PPAR) pathway [62], which has been indicated as a new biomarker of follicular capacity [63]. By constructing a IncRNA-mRNA co-expression network, we found that SLC16A10 was positively regulated by multiple IncRNAs. Additionally, the function of the target gene SLC16A10 was enriched mainly in thyroid hormone synthesis as well as the binding, digestion, and absorption processes of proteins. One retrospective study found that increased levels of thyrotropic hormone may be associated with decreased serum levels of AMH [64], whereas the ovarian reserve was not associated with anti-thyroid peroxidase antibodies or anti-thyroglobulin antibodies. Transplantation of human amniotic epithelial cells has been shown to restore ovarian function and improve ovarian reserve and fertility in cyclophosphamide-induced POI rats by affecting protein digestion/absorption, and steroid-biosynthesis pathways [65].

\section{Conclusions}

The expression profiles of IncRNAs and mRNAs in OGCs from women with DOR and NOR were mined using transcriptome sequencing. A total of 466 IncRNAs and 457 mRNAs were identified to have differential expression in the transcripts. Bioinformatic analysis revealed that most of these IncRNAs and mRNAs were involved in important pathways and biological processes related to cell adhesion, apoptosis, steroid biosynthesis, and the immune system. Dysregulated IncRNAs could serve as diagnostic biomarkers. Further studies should concentrate on the elucidation of specific molecular mechanisms to provide a new understanding for the diagnosis and treatment of DOR.

\section{Methods}

\section{Ethical approvalof the study protocol}


The research protocol was approved by the Reproductive Medicine Ethics Committee of the Affiliated Hospital of Shandong University of Traditional Chinese Medicine (Ref: SZ2018090103). All patients provided written informed consent before sample collection.

\section{Patients and sample collection}

Samples and clinical data were collected at the Center for Integrative Reproduction and Genetics, Affiliated Hospital of Shandong University of Traditional Chinese Medicine, from September 2018 to October 2020. Twenty-four patients were recruited in our study. These patients underwent IVF/ICSI using a gonadotrophin-releasing hormone antagonist for controlled ovarian hyperstimulation. All patients satisfied the following criteria: age of $25-40$ years; body mass index (BMI) of $18-25 \mathrm{~kg} / \mathrm{m}^{2}$; and no endocrine disease, uterine anomaly, endometriosis, or chromosomal abnormalities.

The study group $(n=12)$ comprised infertile women with DOR. The diagnostic criteria adopted an amended version of the Bologna standard [66], with participants fulfilling a minimum of two of the following conditions: (i) bilateral $\mathrm{AFC}<6$; (ii) $\mathrm{AMH}<1.10 \mathrm{ng} / \mathrm{mL}$; (iii) menstrual basal FSH level of 10-40 $\mathrm{mIU} / \mathrm{mL}$. The control group $(\mathrm{n}=12)$ was women with NOR and pure male factor (azoospermia or severe oligospermia/aspermia) infertility. Six patient samples from each group were selected randomly for IncRNA sequencing. The other six samples underwent RT-qPCR.

OGCs were collected and purified by density-gradient centrifugation from follicular fluid, which was obtained by ultrasound-guided transvaginal ovarian puncture for oocyte retrieval from the first aspiration follicle of each ovary. Oocyte retrieval was performed $36 \mathrm{~h}$ after injection of human chorionic gonadotropin once follicles $>17 \mathrm{~mm}$ in diameter. Follicular fluid was centrifuged at $2000 \mathrm{rpm}$ for $5 \mathrm{~min}$ at room temperature and the supernatant was removed. Phosphate-buffered saline $(5 \mathrm{~mL})$ was added to the precipitate and mixed. Then, $5 \mathrm{~mL}$ of Ficoll-Paque (GE Healthcare, Chicago, IL, USA) was added to a 15$\mathrm{mL}$ centrifuge tube. The suspension was added slowly to the upper layer of Ficoll-Paque, and centrifugation at $2000 \mathrm{rpm}$ for $20 \mathrm{~min}$ at room temperature was carried out. The OGC layer was aspirated and transferred to a 1.5-mL centrifuge tube, and repeated pipetting was undertaken followed by centrifugation at $2000 \mathrm{rpm}$ for $3 \mathrm{~min}$ at room temperature. Finally, the supernatant was removed and the OGCs were stored in a $-80^{\circ} \mathrm{C}$ for further analyses.

\section{Preparation and sequencing of RNA libraries}

TRIzol ${ }^{8}$ Reagent (Invitrogen, Carlsbad, CA, USA) was employed to isolate and purify total RNA according to the manufacturer's instructions. The amount and purity of total RNA in each sample was analyzed using a bioanalyzer (2100 series; Agilent Technologies, Santa Clara, CA, USA) and an RNA Nano1000 Assay Kit (Agilent Technologies) so that the RNA integrity number (RIN) > 7.0. Ribosomal RNA was removed according to the instructions of the Ribo-Zero ${ }^{\text {TM }}$ rRNA Removal Kit (Illumina). Under a high temperature, the remaining RNA was broken into short fragments using divalent cations. Subsequently, cDNA was created by reverse transcription using fragmented RNA as a template, followed by addition of 
deoxynucleoside triphosphate, RNase $\mathrm{H}$, and Escherichia coli DNA polymerase I to synthesize the second strand. After purification of AMPure XP beads (Beckman Coulton, Fullerton, CA, USA) and end repair, poly $(A)$ was added, and sequencing connectors were attached. Then, CDNA of a certain length range was extracted. PCR amplification was performed to obtain a cDNA library. The average insert size of the final cDNA library was $300 \pm 50 \mathrm{bp}$. Twelve separate RNA-seq libraries were generated for the study group and control group.

\section{RNA-seq}

High-throughput sequencing of samples was executed on a NovaSeq 6000 using $2 \times 150$-bp paired-end sequences (PE150) according to a standard procedure. First, reads containing adapter contamination, low-quality bases, or unidentified bases were excluded by applying cutadapt v1.9 (https://cutadapt.readthedocs.io/en/stable/\#). Then, the quality of reads was authenticated using FastQC v0.10.1 (www.bioinformatics.babraham.ac.uk/projects/fastqc/). The Q20, Q30, and GC contents of clean data were calculated, and subsequent analyses were based on these data. Sequencing and data acquisition were undertaken by LC-Bio Technology (Hangzhou, China).

\section{Transcript assembly}

HISAT (v2.0.4) was utilized to preprocess raw data and map the processed valid data to the human reference genome (GRCh38). Annotation files in Gene Transfer Format were created to identify which genes these reads mapped to. The mapped reads from each sample were assembled using StringTie v1.3.0 (https://ccb.jhu.edu/software/stringtie/index.shtml/) with default parameters. Then, all transcripts in the sample were joined using gffcompare (https://ccb.jhu.edu/software/stringtie/gffcompare.shtml) to build a whole transcriptome. Then, StringTie was used to calculate fragments per kilobase of exon model per million mapped reads (FPKM) to quantify the expression of the transcripts.

\section{IncRNA identification}

First, transcripts that overlapped with known mRNAs and transcripts shorter than 200 bp were removed. If the remaining transcripts duplicated the IncRNA, then these transcripts were deemed to be "known IncRNAs". The rest of the non-annotated transcripts were assumed to recognize the underlying novel IncRNAs. Then, we employed CPC (0.9-r2) and $\mathrm{CNCl}$ (v2.0) to forecast the coding potential of the remaining transcripts of length $\geq 200 \mathrm{bp}$, mapped read coverage $\geq 3$, and exon number $\geq 1$. Transcripts with a CPC score $<-1$ and $\mathrm{CNCl}$ score $<0$ were assumed to be potential novel IncRNAs.

\section{Differential expression of mRNAs and IncRNAs}

StringTie was adopted to evaluate the expression of mRNAs and IncRNAs by calculation of FPKM. Multiple values were analyzed by the packages -edgeR or DESeq2 within $R(R$ Institute for Statistical Computing, Vienna, Austria). $P<0.05$ and $\mid \log 2$ (fold change) $\mid \geq 1$ were used as the basis for screening DE mRNAs and IncRNAs between the DOR group and NOR group.

\section{Target-gene prediction and functional analyses of IncRNAs}


The interaction between IncRNAs and their adjacent genes is known as "cis-regulation". We used Python scripts to predict the cis-regulatory relationships between mRNAs and IncRNAs in the 100-kbp range upstream and downstream of chromosomes. We undertook analyses of enrichment of the functions and signaling pathways of IncRNA-targeted mRNAs using GO and KEGG databases, respectively.

\section{Validation of IncRNA expression by RT-qPCR}

Based on analysis of sequencing results and literature review, the expression of seven IncRNAs (GNG12, MEG3, NEAT1, MST1L, RAB5A, GCN1 and ZEB2-AS1) was measured by RT-qPCR in OGC samples from 12 patients to validate the RNA-seq results. Total RNA was extracted from samples using the RNeasy Micro Kit following the manufacturer's instructions (Qiagen, Stanford, VA, USA). The content and purity of RNA were measured at an absorbance of $260 / 280 \mathrm{~nm}$ using Scandrop ${ }^{\text {TM }} 100$ (Analytik Jena, Jena, Germany). Total RNA was reverse-transcribed into cDNA using the TUREscript First Strand cDNA Synthesis Kit (Aidlab, Beijing, China) according to the manufacturer's instructions. The specific primers designed for RT-qPCR using Primer-Blast (National Center for Biotechnology Information) and primerprimer6 together are listed in Table 7. The analytikjena-qTOWER2.2 PCR System (Analytik Jena) was applied to conduct RT-qPCR. The reaction protocol was as follows: initial denaturation at $95^{\circ} \mathrm{C}$ for $3 \mathrm{~min}$, denaturing at $95^{\circ} \mathrm{C}$ for $10 \mathrm{~s}$, and annealing at $60^{\circ} \mathrm{C}$ for $30 \mathrm{~s}$ for 40 cycles. The expression of glyceraldehyde-3-phosphate dehydrogenase (an endogenous reference transcript) was used to quantify relative IncRNA expression using the $2^{-\Delta \Delta C t}$ method. For each reaction, three independent biological replicates were employed. 
Table 7

Primer sequences used for RT-qPCR

\begin{tabular}{|ll|}
\hline Primer & Sequence (5'-3') \\
\hline GAPDH-F & TGCACCACCAACTGCTTAGC \\
\hline GAPDH-R & GGCATGGACTGTGGTCATGAG \\
\hline GNG12-F & TCCCTGTGTCTTTCTTCTC \\
\hline GNG12-R & TAGCCTATGTGCCTCTTG \\
\hline MEG3-F & TGGCATAGAGGAGGTGAT \\
\hline NEAT1-F & AGTGTGAGTCCTAGCATTG \\
\hline NEAT1-R & GAACTTCCTCCTCCTAAGC \\
\hline RAB5A-F & CGGTAACGAGTCCATTGTAG \\
\hline RAB5A-R & CCTTGATGAGTGCTGTATCC \\
\hline ZEB2-AS1-F & CTGGATAGCAAAGGACACC \\
\hline ZEB2-AS1-R & GTTTCTTCGCTTCCACCT \\
\hline MST1L-F & GAGCCTGGAAATGGTTGA \\
\hline MST1L-R & AAGTTCTCCTCCAGTTGTG \\
\hline GCN1-F & AAGGGTAGGAAAGTCTGGTTA \\
\hline GCN1-R & ATTGAGAATCGAACCTGTTATCA \\
\hline
\end{tabular}

\section{Statistical analyses}

SPSS 23.0 (IBM, Armonk, NY, USA) was used for statistical analyses. Prism 8.0.1 (GraphPad, San Diego, CA, USA) was employed for statistical analyses of RT-qPCR results and graphs. Measurement data are expressed as the mean \pm SD. Student's $t$ test was undertaken to determine a discrepancy in the expression of IncRNAs between the DOR group and NOR group. $\mathrm{P}<0.05$ was deemed significant.

\section{Abbreviations}

AFC

Antral follicle count

$\mathrm{AMH}$

Anti- Müllerian hormone

BMI

Body Mass Index 
BP

Biological process

CC

Cellular component

cDNA

Complementary DNA

$\mathrm{CNCl}$

Coding-Non-Coding Index

CPC

Coding Potential Calculator

DE

Differentially expressed

DOR

Diminished ovarian reserve

FPKM

Fragments per kilobase of exon model per million mapped reads

$\mathrm{FSH}$

Follicle-stimulating hormone

GO

Gene Ontology

ICSI

Intracytoplasmic sperm injection

IVF

In vitro fertilization

KEGG

Kyoto Encyclopedia of Genes and Genomes

IncRNAs

Long noncoding RNAs

MF

Molecular function

NOR

Normal ovarian reserve

OGCs

Ovarian granulosa cells

ORF

Open reading frame

PCOS

Polycystic ovary syndrome

POF

Premature ovarian failure 
POI

Premature ovarian insufficiency

PPAR

Peroxisome proliferator-activated receptor

RT-qPCR

Reverse transcription-quantitative polymerase chain reaction

RNA-seq

RNA-sequencing.

\section{Declarations}

Ethics approval and consent to participate

The study was conducted in accordance with the Declaration of Helsinki, and approved by the Ethics Committee of the Reproductive Medicine Ethics Committee of the Affiliated Hospital of the Shandong University of Traditional Chinese Medicine (Ref. SZ2018090103). Written informed consent was obtained from all the participants.

Consent for publication

Not applicable.

Availability of Data and materials

All the raw data has been stored in GEO (Gene Expression Omnibus) with the login number GSE193136 (https://www.ncbi.nlm.nih.gov/ geo/query/acc.cgi?acc=GSE193136).

Competing interests

The authors declare that they have no commercial or financial relationships that could be construed as a potential conflict of interest.

Funding

This research was funded by Natural Science Foundation of Shandong Province (NO. ZR2021MH255), National Natural Science Foundation of China (NO. 81703958, 81774355 and 81974577) and Key Technology Research and Development Program of Shandong (NO. 2019GSF108067).

Authors' Contributions

FL and HW conceived and designed this study. LD contributed to the acquisition and analyses of data. LD and XX contributed to drafting the manuscript. CY was responsible for sample collection. HMC and PCKL contributed to manuscript revision. All authors read and approved the final submitted version of this manuscript. 
Acknowledgments

The authors thank the nurses and laboratory staff of the Department of Assisted Reproduction of the Affiliated Hospital of Shandong University of Traditional Chinese Medicine for their technical assistance.

\section{References}

1. Qi Z, Liu Y, Yang H, Yang X, Wang H, Liu B, Yuan Y, Wang G, Xu B, Liu W, Xu Z and Deng Y (2021) Protective role of $\mathrm{mA}$ binding protein YTHDC2 on CCNB2 in manganese-induced spermatogenesis dysfunction. Chemico-biological interactions:109754. doi: 10.1016/j.cbi.2021.109754

2. Navot D, Rosenwaks $Z$ and Margalioth E (1987) Prognostic assessment of female fecundity. Lancet (London, England) 2:645-7. doi: 10.1016/s0140-6736(87)92439-1

3. Devine K, Mumford SL, Wu M, DeCherney AH, Hill MJ and Propst A (2015) Diminished ovarian reserve in the United States assisted reproductive technology population: diagnostic trends among 181,536 cycles from the Society for Assisted Reproductive Technology Clinic Outcomes Reporting System. Fertil Steril 104:612 - 19.e3. doi: 10.1016/j.fertnstert.2015.05.017

4. Balmaceda $\mathrm{J}$ and Schwarze $\mathrm{J}$ (2007) Assessment of ovarian reserve - should we perform tests of ovarian reserve routinely? Human reproduction (Oxford, England) 22:1492; author reply 1492-3. doi: 10.1093/humrep/del509

5. Cohen J, Chabbert-Buffet N and Darai E (2015) Diminished ovarian reserve, premature ovarian failure, poor ovarian responder-a plea for universal definitions. Journal of assisted reproduction and genetics 32:1709-12. doi: 10.1007/s10815-015-0595-y

6. Ponting $C$ and Belgard $T$ (2010) Transcribed dark matter: meaning or myth? Human molecular genetics 19:R162-8. doi: 10.1093/hmg/ddq362

7. Kumar M and Goyal R (2017) LncRNA as a Therapeutic Target for Angiogenesis. Current topics in medicinal chemistry 17:1750-1757. doi: 10.2174/1568026617666161116144744

8. Kong Y, Lu Z, Liu P, Liu Y, Wang F, Liang E, Hou F and Liang M (2019) Long Noncoding RNA: Genomics and Relevance to Physiology. Comprehensive Physiology 9:933-946. doi: $10.1002 /$ cphy.c180032

9. McDonel P and Guttman M (2019) Approaches for Understanding the Mechanisms of Long Noncoding RNA Regulation of Gene Expression. Cold Spring Harbor perspectives in biology 11. doi: 10.1101/cshperspect.a032151

10. Cui D, Ma J, Liu Y, Lin K, Jiang X, Qu Y, Lin J and Xu K (2018) Analysis of long non-coding RNA expression profiles using RNA sequencing in ovarian endometriosis. Gene 673:140-148. doi: 10.1016/j.gene.2018.06.046

11. Pankiewicz K, Laudański P and Issat T (2021) The Role of Noncoding RNA in the Pathophysiology and Treatment of Premature Ovarian Insufficiency. International journal of molecular sciences 22. doi: $10.3390 /$ ijms22179336 
12. Huang $X$, Hao C, Bao H, Wang M and Dai $H$ (2016) Aberrant expression of long noncoding RNAs in cumulus cells isolated from PCOS patients. Journal of assisted reproduction and genetics 33:11121. doi: $10.1007 / \mathrm{s} 10815-015-0630-z$

13. Jiang Z, Jin L, Shi W, Xi J, Hu Y, Liu X, Sheng J, Ding G and Huang H (2019) A combination of follicle stimulating hormone, estradiol and age is associated with the pregnancy outcome for women undergoing assisted reproduction: a retrospective cohort analysis. Science China. Life sciences 62:112-118. doi: 10.1007/s11427-018-9322-6

14. Chen S, Wang Y, Liao L, Meng L, Li J, Shi C, Han H, Zheng X and Shen H (2020) Similar Repair Effects of Human Placenta, Bone Marrow Mesenchymal Stem Cells, and Their Exosomes for Damaged SVOG Ovarian Granulosa Cells. Stem cells international 2020:8861557. doi: 10.1155/2020/8861557

15. Xiong Y, Liu T, Wang S, Chi H, Chen C and Zheng J (2017) Cyclophosphamide promotes the proliferation inhibition of mouse ovarian granulosa cells and premature ovarian failure by activating the IncRNA-Meg3-p53-p66Shc pathway. Gene 596:1-8. doi: 10.1016/j.gene.2016.10.011

16. Yao G, He J, Kong Y, Zhai J, Xu Y, Yang G, Kong D, Dong F, Shi S, Yang Q and Sun Y (2019) Transcriptional profiling of long noncoding RNAs and their target transcripts in ovarian cortical tissues from women with normal menstrual cycles and primary ovarian insufficiency. Molecular reproduction and development 86:847-861. doi: 10.1002/mrd.23158

17. Zhao W and Dong L (2018) Long non-coding RNA HOTAIR overexpression improves premature ovarian failure by upregulating Notch-1 expression. Experimental and therapeutic medicine 16:47914795. doi: $10.3892 /$ etm. 2018.6750

18. Bouckenheimer J, Assou S, Riquier S, Hou C, Philippe N, Sansac C, Lavabre-Bertrand T, Commes T, Lemaître JM, Boureux A and De Vos J (2016) Long non-coding RNAs in human early embryonic development and their potential in ART. Hum Reprod Update 23:19-40. doi:

10.1093/humupd/dmw035

19. Tu J, Chen Y, Li Z, Yang H, Chen H and Yu Z (2020) Long non-coding RNAs in ovarian granulosa cells. J Ovarian Res 13:63. doi: 10.1186/s13048-020-00663-2

20. Zheng Y, Bian Y, Wu R, Chen W, Fu L, Li P, Wang Y, Yang X, Zhao S and Shi Y (2021) High-Throughput Sequencing Profiles About IncRNAs and mRNAs of Ovarian Granulosa Cells in Polycystic Ovary Syndrome. Frontiers in medicine 8:741803. doi: 10.3389/fmed.2021.741803

21. Meng L, Zhao K, Wang CC, Tao J, Wu Z, Teerds K and Zhang S (2021) Characterization of Long NonCoding RNA Profiles in Porcine Granulosa Cells of Healthy and Atretic Antral Follicles: Implications for a Potential Role in Apoptosis. Int J Mol Sci 22. doi: 10.3390/ijms22052677

22. Wang Y, Shi H, Zhang G, Wu P, Chen L, Shen M, Li T, Lv X, Gu Y and Wang J (2021) Transcriptome Analysis of Long Noncoding RNAs and mRNAs in Granulosa Cells of Jinghai Yellow Chickens Illuminated With Red Light. Front Genet 12:563623. doi: 10.3389/fgene.2021.563623

23. Wang L, Fan H, Zou Y, Yuan Q, Hu X, Chen X, Zhu C, Zhang X and Cui H (2020) Aberrant Expression of Long Non-coding RNAs in Exosomes in Follicle Fluid From PCOS Patients. Front Genet 11:608178. doi: $10.3389 /$ fgene.2020.608178 
24. Khalaj K, Miller JE, Lingegowda H, Fazleabas AT, Young SL, Lessey BA, Koti M and Tayade C (2019) Extracellular vesicles from endometriosis patients are characterized by a unique miRNA-IncRNA signature. JCl Insight 4. doi: 10.1172/jci.insight.128846

25. Wu J, Huang $H$, Huang W, Wang L, Xia X and Fang X (2020) Analysis of exosomal IncRNA, miRNA and mRNA expression profiles and ceRNA network construction in endometriosis. Epigenomics 12:1193-1213. doi: 10.2217/epi-2020-0084

26. Guo S, Quan S and Zou S (2021) Roles of the Notch Signaling Pathway in Ovarian Functioning. Reprod Sci 28:2770-2778. doi: 10.1007/s43032-021-00610-6

27. Zheng C, Liu S, Qin Z, Zhang X and Song Y (2021) LncRNA DLEU1 is overexpressed in premature ovarian failure and sponges miR-146b-5p to increase granulosa cell apoptosis. J Ovarian Res 14:151. doi: 10.1186/s13048-021-00905-x

28. Ruszkowska M, Nynca A, Paukszto L, Sadowska A, Swigonska S, Orlowska K, Molcan T, Jastrzebski JP and Ciereszko RE (2018) Identification and characterization of long non-coding RNAs in porcine granulosa cells exposed to 2,3,7,8-tetrachlorodibenzo-p-dioxin. J Anim Sci Biotechnol 9:72. doi: 10.1186/s40104-018-0288-3

29. Majewska M, Lipka A, Paukszto L, Jastrzebski JP, Gowkielewicz M, Jozwik M and Majewski MK (2018) Preliminary RNA-Seq Analysis of Long Non-Coding RNAs Expressed in Human Term Placenta. Int J Mol Sci 19. doi: 10.3390/ijms19071894

30. Dang Y (2016) The role and mechanism of non-coding RNA in primary ovarian insufficiency. Ph.D. thesis. Shandong University.

31. Li D, Wang X, Dang Y, Zhang X, Zhao S, Lu G, Chan WY, Leung PCK and Qin Y (2021) IncRNA GCAT1 is involved in premature ovarian insufficiency by regulating p27 translation in GCs via competitive binding to PTBP1. Mol Ther Nucleic Acids 23:132-141. doi: 10.1016/j.omtn.2020.10.041

32. Li D, Xu W, Wang X, Dang Y, Xu L, Lu G, Chan WY, Leung PCK, Zhao S and Qin Y (2021) IncRNA DDGC participates in premature ovarian insufficiency through regulating RAD51 and WT1. Mol Ther Nucleic Acids 26:1092-1106. doi: 10.1016/j.omtn.2021.10.015

33. Wang F, Chen X, Sun B, Ma Y, Niu W, Zhai J and Sun Y (2021) Hypermethylation-mediated downregulation of IncRNA PVT1 promotes granulosa cell apoptosis in premature ovarian insufficiency via interacting with Foxo3a. J Cell Physiol 236:5162-5175. doi: 10.1002/jcp.30222

34. Harvey CN, Esmail M, Wang Q, Brooks Al, Zachow R and Uzumcu M (2009) Effect of the methoxychlor metabolite HPTE on the rat ovarian granulosa cell transcriptome in vitro. Toxicol Sci 110:95-106. doi: 10.1093/toxsci/kfp089

35. Wang $X$, Meng $K$, Wang H, Wang $Y$, Zhao Y, Kang J, Zhang $Y$ and Quan $F$ (2021) Identification of small extracellular vesicle subtypes in follicular fluid: Insights into the function and miRNA profiles. $J$ Cell Physiol 236:5633-5645. doi: 10.1002/jcp.30251

36. Chen $Y$, Chang H, Cheng J, Tsai H, Wu C and Leung P (2015) Transforming growth factor- $\beta 1$ upregulates connexin43 expression in human granulosa cells. Human reproduction (Oxford, England) 30:2190-201. doi: 10.1093/humrep/dev175

Page 22/30 
37. Craig J, Orisaka M, Wang H, Orisaka S, Thompson W, Zhu C, Kotsuji F and Tsang B (2007) Gonadotropin and intra-ovarian signals regulating follicle development and atresia: the delicate balance between life and death. Frontiers in bioscience: a journal and virtual library 12:3628-39. doi: $10.2741 / 2339$

38. Wang Y, Hu SB, Wang MR, Yao RW, Wu D, Yang L and Chen LL (2018) Genome-wide screening of NEAT1 regulators reveals cross-regulation between paraspeckles and mitochondria. Nat Cell Biol 20:1145-1158. doi: 10.1038/s41556-018-0204-2

39. Bouckenheimer J, Fauque P, Lecellier CH, Bruno C, Commes T, Lemaître JM, De Vos J and Assou S (2018) Differential long non-coding RNA expression profiles in human oocytes and cumulus cells. Sci Rep 8:2202. doi: 10.1038/s41598-018-20727-0

40. Ernst EH, Nielsen J, Ipsen MB, Villesen P and Lykke-Hartmann K (2018) Transcriptome Analysis of Long Non-coding RNAs and Genes Encoding Paraspeckle Proteins During Human Ovarian Follicle Development. Front Cell Dev Biol 6:78. doi: 10.3389/fcell.2018.00078

41. Zhen J, Li J, Li X, Wang X, Xiao Y, Sun Z and Yu Q (2021) Downregulating IncRNA NEAT1 induces proliferation and represses apoptosis of ovarian granulosa cells in polycystic ovary syndrome via microRNA-381/IGF1 axis. J Biomed Sci 28:53. doi: 10.1186/s12929-021-00749-z

42. Ma R, Hou X, Zhang L, Sun SC, Schedl T, Moley K and Wang Q (2014) Rab5a is required for spindle length control and kinetochore-microtubule attachment during meiosis in oocytes. Faseb j 28:402635. doi: $10.1096 / \mathrm{fj} .14-250886$

43. Xiang Z, Lv Q, Chen X, Zhu X, Liu S, Li D and Peng X (2020) Lnc GNG12-AS1 knockdown suppresses glioma progression through the AKT/GSK-3 $\beta / \beta$-catenin pathway. Biosci Rep 40. doi:

$10.1042 /$ bsr20201578

44. Mahboobeh Z, Pegah M, Fatemeh S, Elham K, Hanieh A, Milad R and Mohammad S (2020) IncRNA ZEB2-AS1: A promising biomarker in human cancers. IUBMB Life 72:1891-1899. doi: 10.1002/iub.2338

45. Zhang B, Fan DB, Liu L, Qin Y and Feng DQ (2020) Knockdown of ZEB2-AS1 inhibits cell proliferation, invasion and induces apoptosis in osteosarcoma by combining with EZH2. Eur Rev Med Pharmacol Sci 24:6533-6539. doi: 10.26355/eurrev_202006_21637

46. Sun F, Tan W, Dan Y, Wang X, Guo Y and Deng G (2020) Copy number gain of pro-inflammatory genes in patients with HBV-related acute-on-chronic liver failure. BMC Med Genomics 13:180. doi: 10.1186/s12920-020-00835-5

47. Guerrero-Netro HM, Chorfi Y and Price CA (2015) Effects of the mycotoxin deoxynivalenol on steroidogenesis and apoptosis in granulosa cells. Reproduction 149:555-61. doi: 10.1530/rep-150018

48. Wang H, He Y, Cheng D, Pu D, Tan R, Gao L, Cui Y and Wu J (2019) Cypermethrin exposure reduces the ovarian reserve by causing mitochondrial dysfunction in granulosa cells. Toxicol Appl Pharmacol 379:114693. doi: 10.1016/j.taap.2019.114693 
49. Ożegowska K, Brązert M, Ciesiółka S, Nawrocki MJ, Kranc W, Celichowski P, Jankowski M, Bryja A, Jeseta M, Antosik P, Bukowska D, Skowroński MT, Bruska M, Pawelczyk L, Zabel M, Nowicki M and Kempisty B (2019) Genes Involved in the Processes of Cell Proliferation, Migration, Adhesion, and Tissue Development as New Potential Markers of Porcine Granulosa Cellular Processes In Vitro: A Microarray Approach. DNA Cell Biol 38:549-560. doi: 10.1089/dna.2018.4467

50. Kranc W, Brązert M, Budna J, Celichowski P, Bryja A, Nawrocki MJ, Ożegowska K, Jankowski M, Chermuła B, Dyszkiewicz-Konwińska M, Jeseta M, Pawelczyk L, Bręborowicz A, Rachoń D, Bruska M, Nowicki M, Zabel M and Kempisty B (2019) Genes responsible for proliferation, differentiation, and junction adhesion are significantly up-regulated in human ovarian granulosa cells during a long-term primary in vitro culture. Histochem Cell Biol 151:125-143. doi: 10.1007/s00418-018-1750-1

51. PashaiasI $M$, Ebrahimi $M$ and Ebrahimie $E$ (2016) Identification of the key regulating genes of diminished ovarian reserve (DOR) by network and gene ontology analysis. Mol Biol Rep 43:923-37. doi: $10.1007 /$ s11033-016-4025-8

52. Esmaeili-Fard SM, Gholizadeh M, Hafezian SH and Abdollahi-Arpanahi R (2021) Genome-wide association study and pathway analysis identify NTRK2 as a novel candidate gene for litter size in sheep. PLoS One 16:e0244408. doi: 10.1371/journal.pone.0244408

53. Qazi IH, Cao Y, Yang H, Angel C, Pan B, Zhou G and Han H (2021) Impact of Dietary Selenium on Modulation of Expression of Several Non-Selenoprotein Genes Related to Key Ovarian Functions, Female Fertility, and Proteostasis: a Transcriptome-Based Analysis of the Aging Mice Ovaries. Biol Trace Elem Res 199:633-648. doi: 10.1007/s12011-020-02192-x

54. Yang X, Zhou Y, Peng S, Wu L, Lin HY, Wang S and Wang H (2012) Differentially expressed plasma microRNAs in premature ovarian failure patients and the potential regulatory function of mir-23a in granulosa cell apoptosis. Reproduction 144:235-44. doi: 10.1530/rep-11-0371

55. Kirshenbaum M and Orvieto R (2019) Premature ovarian insufficiency (POI) and autoimmunity-an update appraisal. J Assist Reprod Genet 36:2207-2215. doi: 10.1007/s10815-019-01572-0

56. Sharif K, Watad A, Bridgewood C, Kanduc D, Amital H and Shoenfeld $Y$ (2019) Insights into the autoimmune aspect of premature ovarian insufficiency. Best Pract Res Clin Endocrinol Metab 33:101323. doi: 10.1016/j.beem.2019.101323

57. Lim W, Bae H, Bazer FW and Song G (2018) Cell-specific expression and signal transduction of C-C motif chemokine ligand 2 and atypical chemokine receptors in the porcine endometrium during early pregnancy. Dev Comp Immunol 81:312-323. doi: 10.1016/j.dci.2017.12.020

58. Matsuda F, Inoue N, Manabe N and Ohkura S (2012) Follicular growth and atresia in mammalian ovaries: regulation by survival and death of granulosa cells. J Reprod Dev 58:44-50. doi: 10.1262/jrd.2011-012

59. Fan Y, Chang Y, Wei L, Chen J, Li J, Goldsmith S, Silber S and Liang X (2019) Apoptosis of mural granulosa cells is increased in women with diminished ovarian reserve. J Assist Reprod Genet 36:1225-1235. doi: 10.1007/s10815-019-01446-5 
60. Hall SE, Upton RMO, McLaughlin EA and Sutherland JM (2018) Phosphoinositide 3-kinase/protein kinase B (PI3K/AKT) and Janus kinase/signal transducer and activator of transcription (JAK/STAT) follicular signalling is conserved in the mare ovary. Reprod Fertil Dev 30:624-633. doi: $10.1071 /$ rd17024

61. Frost ER, Ford EA, Peters AE, Reed NL, McLaughlin EA, Baker MA, Lovell-Badge R and Sutherland JM (2020) Signal transducer and activator of transcription (STAT) 1 and STAT3 are expressed in the human ovary and have Janus kinase 1-independent functions in the COV434 human granulosa cell line. Reprod Fertil Dev 32:1027-1039. doi: 10.1071/rd20098

62. Ding N, Harlow SD, Randolph JF, Jr., Loch-Caruso R and Park SK (2020) Perfluoroalkyl and polyfluoroalkyl substances (PFAS) and their effects on the ovary. Hum Reprod Update 26:724-752. doi: 10.1093/humupd/dmaa018

63. Tatone C, Benedetti E, Vitti M, Di Emidio G, Ciriminna R, Vento ME, Cela V, Borzì P, Carta G, Lispi M, Cimini AM and Artini PG (2016) Modulating Intrafollicular Hormonal Milieu in Controlled Ovarian Stimulation: Insights From PPAR Expression in Human Granulosa Cells. J Cell Physiol 231:908-14. doi: $10.1002 / j c p .25182$

64. Osuka S, Iwase A, Goto M, Takikawa S, Nakamura T, Murase T, Kato N, Kotani T and Kikkawa F (2018) Thyroid Autoantibodies do not Impair the Ovarian Reserve in Euthyroid Infertile Women: A Cross-Sectional Study. Horm Metab Res 50:537-542. doi: 10.1055/a-0637-9430

65. Zhang Y, Ouyang X, You S, Zou H, Shao X, Zhang G, Zhang C and Hu L (2020) Effect of human amniotic epithelial cells on ovarian function, fertility and ovarian reserve in primary ovarian insufficiency rats and analysis of underlying mechanisms by mRNA sequencing. Am J Transl Res 12:3234-3254.

66. Ferraretti AP, La Marca A, Fauser BCJM, Tarlatzis B, Nargund G and Gianaroli L (2011) ESHRE consensus on the definition of 'poor response' to ovarian stimulation for in vitro fertilization: the Bologna criteria. Human Reproduction 26:1616-1624. doi: 10.1093/humrep/der092

\section{Figures}



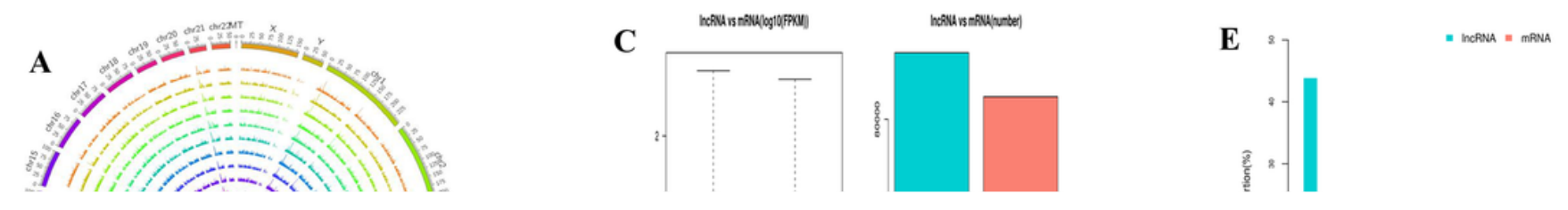

\section{Figure 1}

Comparison of the properties of mRNA and IncRNA from women with NOR or DOR. a Genomic mapping of IncRNAs in different samples. b Location of IncRNA types on 46 chromosomes. Each circle represents a type of IncRNA and corresponds to "i", "j", "o", "u", or " $\mathrm{x}$ " from the outer circle to the inner circle. c The expression and amount of IncRNAs and mRNAs. $\mathbf{d}$ Distribution of the transcript length of IncRNAs and mRNAs. e Distribution of the exon number of IncRNAs and mRNAs. f Distribution of the ORF length of IncRNAs and mRNAs 
A Difterentially expressed genes in different groups

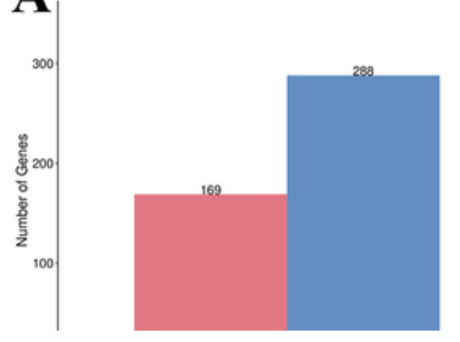

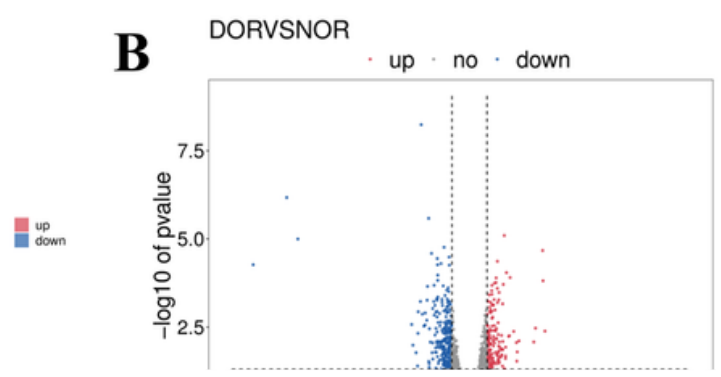
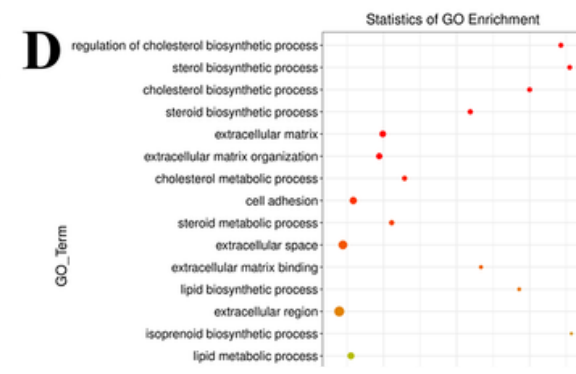

战

\section{Figure 2}

Identification and characterization of the DE mRNA genes between women in the DOR group and NOR group. a Number of DE mRNA genes showing upregulated and downregulated expression. $\mathbf{b}$ Volcano plot of the DE mRNA genes. $\mathbf{c}$ Histogram of the GO-enriched terms of DE mRNA genes. $\mathbf{d}$ Scatter plot of the GO-enriched DE mRNA genes. e Scatter plot of the signaling pathway enrichment of DE mRNA genes using the KEGG database 

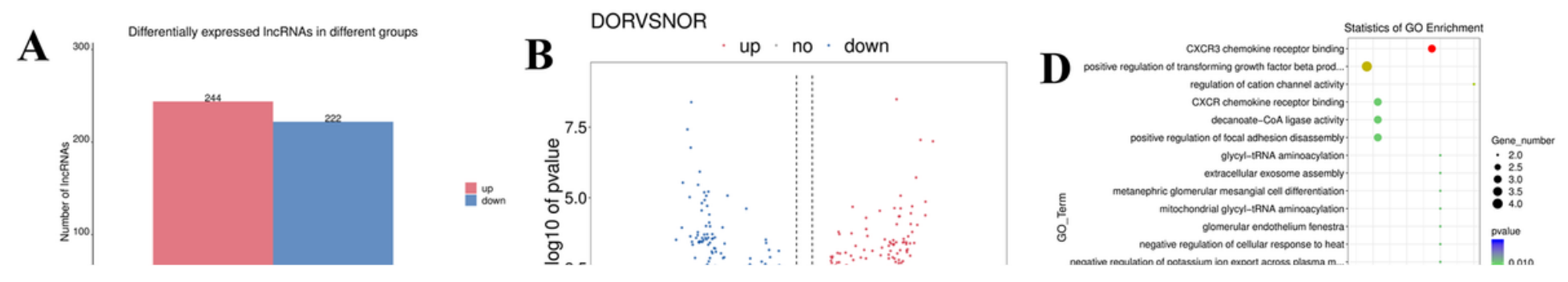

\section{Figure 3}

Identification and characterization of the DE IncRNAs between women in the DOR group and NOR group. a Number of DE IncRNAs with upregulated and downregulated expression. b Volcano plot of the DE IncRNAs. c Histogram of the GO-enriched terms for the target genes of the DE IncRNAs. $\mathbf{d}$ Scatter plot of the $\mathrm{GO}$ enrichment for the target genes of the DE IncRNAs. e Scatter plot of the signaling pathway enrichment for the target genes of the DE IncRNAs using the KEGG database 


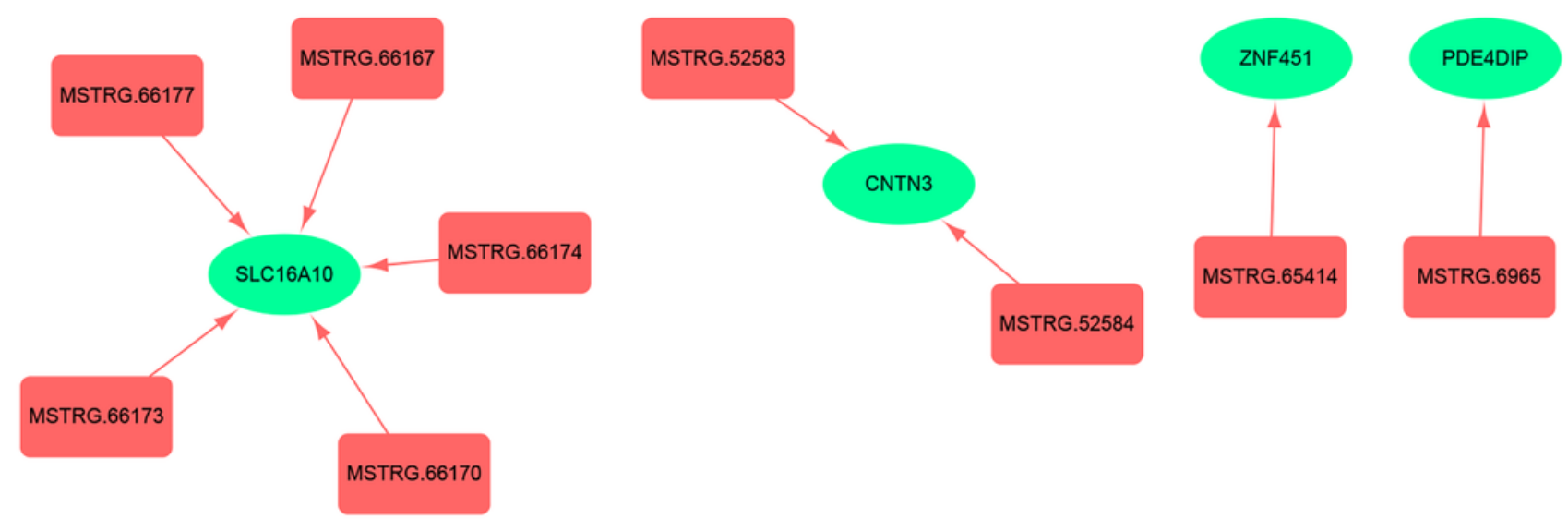

\section{Figure 4}

The IncRNA-mRNA regulatory network. Each IncRNA is a red round rectangle. Each mRNA is a green ellipse 


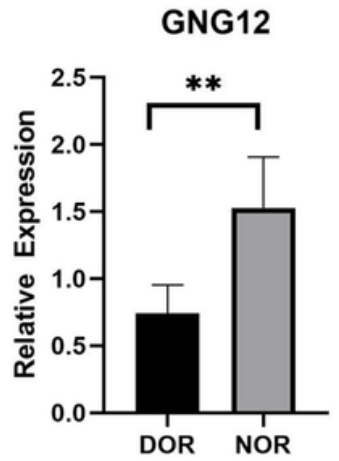

GCN1

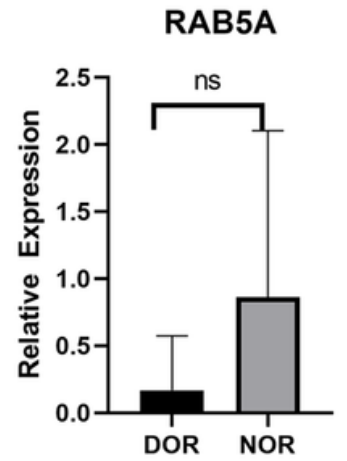

MST1L
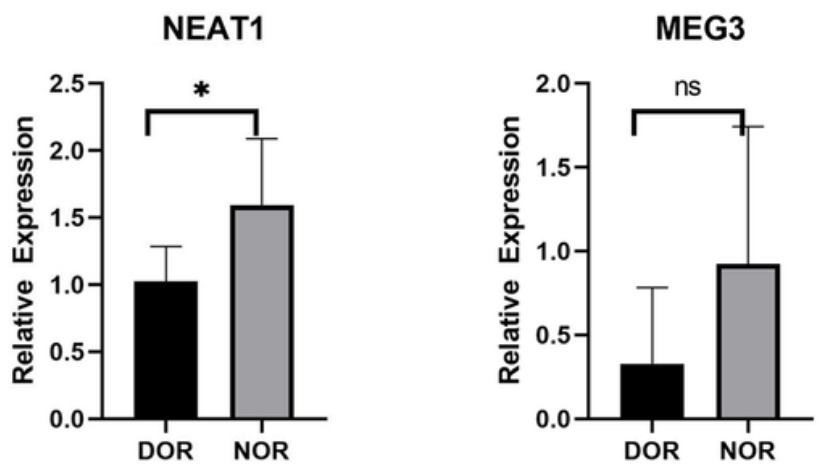

\section{Figure 5}

Expression of key IncRNAs as determined by RT-qPCR. ${ }^{\star} \mathrm{P}<0.05$; ${ }^{\star \star} \mathrm{P}<0.01$

\section{Supplementary Files}

This is a list of supplementary files associated with this preprint. Click to download.

- supplementaryfile.zip 